\title{
Prevalence of Chronic Kidney Disease among Hypertensive non-Diabetic Patients Attending Primary Healthcare Centers in Cairo, Egypt
}

\author{
Silvana Nader Nagib ${ }^{1}$, Saeid Abdel Wahab Afify ${ }^{1}$, Ghada Essam Aldin Amin ${ }^{1}$, and \\ Mohamed Allam ${ }^{1}$ \\ ${ }^{1}$ Ain Shams University Faculty of Medicine
}

November 20, 2020

\begin{abstract}
Background: Although chronic kidney disease (CKD) is considered the major cause of morbidity and mortality in hypertension, the recognition and prevention of CKD remain deficient. Chronic Kidney Disease (CKD) is one of the major health challenges in Egypt. Chronic kidney disease (CKD) affects approximately 13\% of the adult population, resulting in significant morbidity, mortality and health care costs. Patients with more progressive stage 3 or stage 4 CKD experience a high rate of cardiovascular events and death compared to earlier stages of CKD. Aim: This study was performed to determine the prevalence of chronic kidney disease among hypertensive non-diabetics patients attending Primary health care (PHC) Centers in Cairo. Methodology: The study type is a cross sectional study, Study setting: Two Primary Health Care centers (PHCs):Saraya El-kobba and ElSharabya. Sampling method: Recruitment of participants was done in one day weekly. Any known essential hypertensive patients aged 18 or more registered in the two PHC Centers in Cairo. Results: The prevalence of CKD was $33 \%$ among the hypertensive non-diabetic patients. Among CKD participants, the prevalence is more common in females (59.7\%) than males (40.3\%), in those who completed primary education and the illiterates and low socioeconomic class. Surprisingly, it is more common in patients with positive family history of CKD and patients with ischemic heart disease and the antihypertensive drugs used. Conclusion: CKD has a high prevalence among hypertensive non-diabetic patients, and it has a significant morbidity and mortality among those patients.
\end{abstract}

\section{Hosted file}

Prevalence of CKD among Hypertensive Non-Diabetics - IJCP MS.pdf available at https: //authorea.com/users/377464/articles/494135-prevalence-of-chronic-kidney-disease-amonghypertensive-non-diabetic-patients-attending-primary-healthcare-centers-in-cairo-egypt

\section{Hosted file}

Prevalence of CKD among Hypertensive Non-Diabetics - IJCP Tables.pdf available at https: //authorea.com/users/377464/articles/494135-prevalence-of-chronic-kidney-disease-amonghypertensive-non-diabetic-patients-attending-primary-healthcare-centers-in-cairo-egypt 